\title{
Análise das componentes da prova como ponto de partida para a definição de objectivos na natação na categoria de cadetes
}

\author{
António Silva ${ }^{1,2}$ \\ Filipa Silva ${ }^{1}$ \\ António Reis 1 \\ Victor Reis ${ }^{1}$ \\ Daniel Marinho ${ }^{1}$ \\ André Carneiro ${ }^{1}$ \\ Felipe Aidar ${ }^{1}$
}

https://doi.org/10.5628/rpcd.07.02.189

\author{
${ }^{1}$ Departamento de Desporto \\ Universidade de Trás-os-Montes e Alto Douro \\ Vila Real \\ Portugal \\ ${ }_{2}$ Centro de Estudos de Tecnologia, Ambiente e Vida \\ (CETAV) \\ Vila Real \\ Portugal
}

\section{RESUMO}

Os objectivos do presente trabalho foram: (i) determinar as expressões preditivas para as diferentes variáveis cronométricas para os 400 e os 200 metros livres e para os 100 metros mariposa, costas, bruços e livres; por género e por prova; (ii) desenvolver normativas para cada uma das variáveis cronométricas estudadas, por género e por prova; (iii) operacionalizar um modelo para a definição de objectivos do processo, de acordo com os dois objectivos anteriores. Para o efeito, foram estudados 446 nadadores ( 230 do sexo feminino e 216 do sexo masculino) portugueses da categoria cadetes. Através da estatística dedutiva foi possível determinar os valores de A (declive) e de B (ordenada na origem), de forma a construir as equações de regressão lineares para o Tempo de Partida (TP), Tempo de Viragem (TV), Tempo de Chegada (TChg) e Tempo de Nado (TN), nas diferentes provas. As principais conclusões do estudo foram: (i) todas as variáveis cronométricas obtiveram relações estatisticamente significativas com o Tempo Total de Prova (TTP) e foram estabelecidas as respectivas expressões de predição de rendimento; (ii) com o aumento da distância de prova houve uma diminuição da importância relativa do TP e um aumento da importância relativa do TV; (iii) para a generalidade das provas estudadas, os nadadores do G5/G6 masculino são mais rápidos a partir, a virar e a chegar do que as nadadoras pertencentes ao G4/G5 feminino.

Palavras-chave: natação, análise cronométrica, definição de objectivos

\begin{abstract}
Chronometric analyses of swimming events in infantile swimmers: An objective model for goal setting

The aims of the present work were: (i) to determine for each gender predictive equations for the different chronometric variables in 400 and $200 \mathrm{~m}$ freestyle and in $100 \mathrm{~m}$ butterfly, backstroke, breaststroke and freestyle; (ii) to develop models for each of the chronometric variables that were studied in the two genders; (iii) to construct an objective model for goal setting. For this purpose, 446 Portuguese swimmers (230 female and 216 male) belonging to the infant age group category were studied. Through deductive statistic procedures it was possible to determine linear regression equations to predict the Starting Time (ST), the Turning Time (TT), the Finishing Time (FT) and the Swimming Time (SwT) in each studied event. The major findings of this study were: (i) all the chronometric variables were significantly related to Total Race Time and the respective performance predictive equations were established; (ii) with the increase of the race distance a decrease in the relative importance of $S T$ and an increase in the relative importance of TT were observed; (iii) to the majority of the studied events, male swimmers were faster to start, to turn and to finish than female swimmers.
\end{abstract}

Key-words: swimming, chronometric analyses, goal setting 


\section{INTRODUÇÃO}

Programar significa sistematizar os conteúdos do processo de treino, segundo objectivos, bem definidos, da preparação de um atleta e dos princípios específicos que determinam a forma racional de organização das cargas de treino (25) num período de tempo bem definido (28). Com o objectivo de elevar ao máximo a prestação do atleta, maximizar o seu rendimento desportivo e consolidar essas melhorias, torna-se necessário ao treinador observar o comportamento do seu atleta e/ou equipa em competição (33), de modo a obter os instrumentos necessários para o treino específico, permitindo assim: (i) elaborar simuladores com tempos parciais, frequências e distâncias de ciclo específicas da competição; (ii) comparar os tempos individuais de partida e viragem com os tempos realizados em competição e com os valores da norma (8, 22); (iii) determinar o número de repetições para o treino da força-resistência, especialmente nos desportos cíclicos.

Actualmente, em natação, existem várias metodologias de observação da competição que avaliam o desempenho do nadador (27). Cada metodologia ou sistema de análise orienta-se segundo um protocolo de observação, de forma a determinar parâmetros cinemáticos julgados convenientes para avaliar o comportamento e desempenho do nadador (33). As competições constituem um terreno de observação privilegiado, que não pode ser nem minimizado nem negligenciado na análise dos factores que concorrem para a optimização da prestação desportiva (24). É possível e desejável recolher certas informações relativas ao desenrolar cronológico e técnico de diferentes provas $(10,11,13,18,21,32)$.

Segundo Reischle (21), para se poder treinar especificamente para a competição são necessários os dados obtidos nesta. Dois nadadores podem realizar o mesmo tempo em determinada prova, mas os meios, as soluções motoras, tácticas, técnicas, etc., podem ser diametralmente opostas. É difícil quantificar de que modo os vários factores da prova sobressaem relativamente a outros.

No entanto, Haljand e Saagpakk (14) referem que, quando se pretende realizar a análise da competição, tem que se decompô-la nos seus elementos constituintes. Assim sendo, entendemos que a análise da competição consiste em medir as diferentes compo- nentes do tempo total de prova, ou seja, o tempo de partida (TP), o tempo de nado (TN), o tempo de viragem (TV) e o tempo de chegada (TChg). Neste sentido, e sempre tendo como base estas quatro componentes de prova, diversos foram os estudos efectuados até à data. Arellano (4), no seu estudo sobre a variabilidade entre as variáveis cronométricas, concluiu que: (i) a importância da partida e da chegada diminui percentualmente à media que a distância de prova aumenta; (ii) a importância do tempo relativo de viragem aumenta à medida que aumenta a distância das provas; e (iii) o tempo relativo de nado aumenta à medida que a distância da prova aumenta.

Arellano et al. (4), nos seus estudos sobre as provas de 50 e 100 metros dos Jogos Olímpicos de 1992, obtiveram coeficientes de correlação e equações de predição lineares, como sendo o resultado de a (declive) e b (ordenada na origem). O tipo de equações que obtiveram foi (equação 1):

Componente da Prova $=(\mathrm{a}$ x tempo da prova $)+\mathrm{b}$ (equação 1)

Neste sentido, o problema central deste trabalho reside na normalização de cada uma das componentes da prova do calendário nacional português para nadadores da categoria cadetes face ao Tempo Total de Prova (TTP), de forma a poder ser utilizado como um meio indispensável para a definição de objectivos de processo, com implicações na programação e execução do programa de treino à qual o nadador está submetido. Assim, o propósito deste trabalho foi o de: (i) determinar as expressões preditivas para as diferentes variáveis cronométricas, tendo em conta o conhecimento do TTP para os 400 e os 200 metros livres e para os 100 metros mariposa, costas, bruços e livres; por género e por prova; (ii) desenvolver normativas para cada uma das variáveis cronométricas estudadas, por género e por prova; (iii) operacionalizar um modelo para a definição de objectivos orientado para a prestação, de acordo com os dois objectivos anteriores.

\section{MATERIAL E MÉTODOS \\ Amostra}

Participaram neste estudo 446 nadadores portugueses (230 do sexo feminino e 216 do sexo masculino) 
da categoria cadetes (10-11 anos nadadoras e 11-12 anos nadadores), que participaram no Torneio Regional da Associação de Natação do Norte de Portugal (ANNP) de piscina curta.

No Quadro 1 apresentamos a distribuição absoluta de casos observados por prova e por género.

Quadro 1. Distribuição absoluta do número de nadadores por género pelas diferentes provas em estudo, que fazem parte da amostra.

\begin{tabular}{lcccccc}
\hline & $\begin{array}{c}400 \mathrm{~m} \\
\text { Livres }\end{array}$ & $\begin{array}{c}200 \mathrm{~m} \\
\text { Livres }\end{array}$ & $\begin{array}{c}100 \mathrm{~m} \\
\text { Livres }\end{array}$ & $\begin{array}{c}100 \mathrm{~m} \\
\text { Bruços }\end{array}$ & $\begin{array}{c}100 \mathrm{~m} \\
\text { Mariposa }\end{array}$ & $\begin{array}{c}100 \mathrm{~m} \\
\text { Costas }\end{array}$ \\
\hline Femininos & 40 & 35 & 38 & 52 & $18^{*}$ & 47 \\
Masculinos & 31 & 14 & 34 & 57 & $23^{*}$ & 57 \\
\hline
\end{tabular}

* O número de atletas na prova de 100 m Mariposa foi menor que nas demais provas corroborando para estudo anterior feito por Barbosa et al. (7) onde o número de nadadores de mariposa foi menor que os demais estilos.

\section{Metodologia}

Procedimentos para captação e registo de imagens

A recolha de imagens para posterior tratamento realizou-se numa piscina de 25,0 x 12,5 x 2,0 metros, e com água à temperatura ambiente. Os registos de vídeo dos nadadores foram feitos em simultâneo através da colocação de três sistemas de captação de imagens aéreas (JVC-SVHS). Todas as câmaras foram apoiadas em suportes de fixação vertical (tripés) e sincronizadas em tempo real com uma mesa de mistura (Panasonic WJMX50). Os sistemas foram colocados na parte superior das bancadas destinadas ao público, no prolongamento de uma linha relativa a um par de marcas de referência, colocadas nas paredes laterais da piscina, que permitiram o registo de imagens aéreas dos nadadores. Estas marcas foram colocadas, paralelamente, aos 7,5m de cada um dos lados da piscina.

Os sistemas de captação de imagem n..$^{\circ} 1$ e n..$^{\circ} 3$ permitiram integrar respectivamente no campo de captação a totalidade do corpo do nadador durante a realização da partida ( $7,5 \mathrm{~m}$ iniciais - sistema de captação n. $\left.{ }^{\circ} 1\right)$, a viragem (7,5 $\mathrm{m}$ de aproximação e 7,5 m de separação - sistemas de captação n. ${ }^{\circ} 1$ e 3 ) e a chegada (últimos $5 \mathrm{~m}$ - sistema de captação n. $\left.{ }^{\circ} 1\right)$. O sistema de captação de imagem $n .{ }^{\circ} 2$, por sua vez, permitiu integrar no campo de captação a totalidade do corpo do nadador durante a realização do percurso central da piscina (distância entre os 7,5 metros iniciais e os 7,5 metros finais) (Figura 1). Todas as câmaras, foram sincronizadas utilizando um sistema visual (foco) tradicional, visível por todas as câmaras.

\section{Procedimentos após a realização da prova}

As características cinemáticas dos registos de imagem efectuados foram determinadas a partir da introdução de um cronómetro no filme e utilizando o dispositivo de "frame by frame", para a cronometragem dos diversos tempos efectuada. Foi considerado como referencial anatómico a passagem da cabeça, porção Frontal (vertex), do nadador por uma linha vertical traçada no ecrã, desde a base de uma marca de referência até à outra (29).

A velocidade média de nado foi deduzida a partir do desenvolvimento da diferença expressa pela equação 2 .

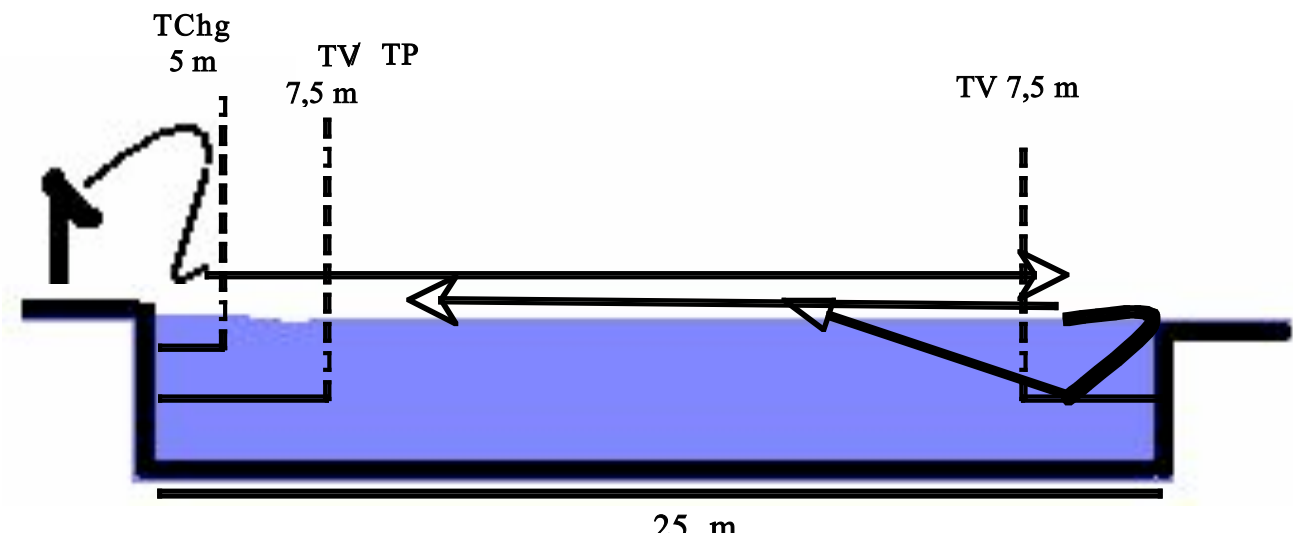

Figura 1. llustração Esquemática do Modelo de Observação (TChg - Tempo de chegada, TV - Tempo de viragem, TP - Tempo de partida). 


$$
\underline{\vec{V}}\left(m s^{-1}\right)=T T P-\left(T P_{\mathrm{i}}+1 \sum_{i=1}^{n} T V_{i}\right)
$$

(equação 2)

\section{Redução dos resultados dos dados. obtidos pelo software de análise cronométrica}

A redução dos dados, obtidos pelo software adobe premier, 2006, para parâmetros estatísticos de tendência central, foi efectuada em folha de cálculo "Excel". Nesta folha de cálculo foram introduzidos os valores absolutos de tempo em cada instante de avaliação. A diferença entre os diferentes instantes consecutivos, foram calculados obtendo assim os valores de cada uma das componentes da prova. Estes valores foram utilizados para o cálculo dos parâmetros normativos desejados.

\section{Procedimentos estatísticos}

Com o intuito de avaliar as associações entre as variáveis em estudo, efectuámos uma análise exploratória das matrizes de correlação. Para o efeito, recorremos aos coeficientes de correlação simples de Pearson (r), para a totalidade das variáveis. A porção de variância comum, associada a ambas as variáveis, foi avaliada pelo coeficiente de determinação $\left(r^{2}\right.$ ajustado). O nível de significância foi mantido em $5 \%$. Através da estatística dedutiva foi possível determinar os valores de B (declive) e de A (ordenada na origem), de forma a ser possível construir as equações de regressão lineares para o TP, TV, TChg e $\mathrm{TN}$, nas diferentes provas (20).

O Quadro 2 pretende apresentar o conjunto de parâmetros analisados.

Quadro 2. Parâmetros cronométricos globais, determinados a partir dos procedimentos de software de analise [adaptado de Vicente (17)].

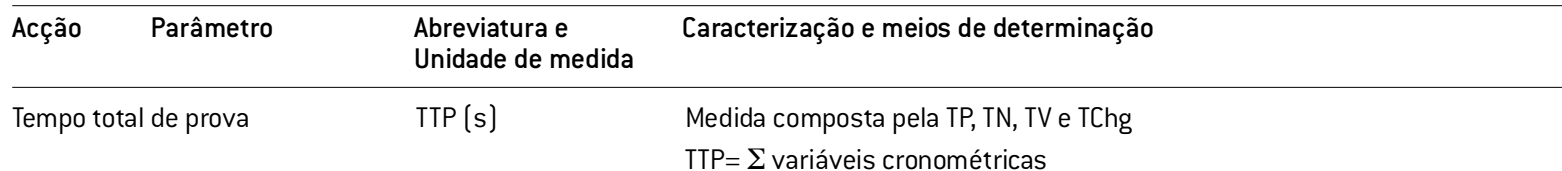

Valor determinado a partir do tempo cronometrado, automaticamente e equivale ao somatório das variáveis cronométricas utilizadas [11].

\begin{tabular}{ll}
\hline Partida Tempo de partida $\quad$ TP $[\mathrm{s}]$ & Valor determinado a partir do tempo cronometrado, automaticamente, desde \\
& o sinal de partida até que a cabeça do nadador passe uma marca colocada a \\
& 7,5 metros do topo da parede onde se encontram os blocos de partida (4). \\
\hline
\end{tabular}

Viragem Tempo de viragem TV $[\mathrm{s}$ )

Valor determinado a partir do tempo cronometrado, automaticamente, para percorrer os 7,5 metros antes da parede (tempo de aproximação) e os 7,5 metros depois (tempo de separação), medido a partir da cabeça do nadador $(2,4,15,17)$.

Chegada Tempo de chegada TChg (s)
percorrer os últimos $5 \mathrm{~m}$, medido a partir do momento que a cabeça do nadador passa esta linha (15).

Nado Tempo de nado TN (s)


Quadro 3. Média $(\bar{x}$ ) e respectivos desvios padrão (DP] de cada uma das componentes cronométricas da prova dos $100 \mathrm{~m}$ mariposa, costas, bruços e livres; para a categoria G4/G5 feminino. M - mariposa; C - costas; $B$ - bruços; $L$ - livres; TTP - tempo total de prova; TP - tempo de partida; TV - tempo de viragem; TChg tempo de chegada; TN - tempo de nado; \% - percentagem relativa do tempo total que os nadadores demoram a cumprir determinada componente da prova.

\begin{tabular}{|c|c|c|c|c|c|c|c|c|c|c|}
\hline \multicolumn{11}{|c|}{ G4/G5 - FEMININO } \\
\hline Prova & TTP & & TP & & TV & & TChg & & TN & \\
\hline $100 \mathrm{~m}$ & $\overline{\mathrm{X}} \pm \mathrm{DP}$ & $\%$ & $\overline{\mathrm{X}} \pm \mathrm{DP}$ & $\%$ & $\bar{X} \pm D P$ & $\%$ & $\overline{\mathrm{X}} \pm \mathrm{DP}$ & $\%$ & $\overline{\mathrm{X}} \pm \mathrm{DP}$ & $\%$ \\
\hline M & $101.18 \pm 10.56$ & 100 & $4.45 \pm 0.58$ & 4.39 & $44.92 \pm 4.68$ & 44.40 & $5.62 \pm 0.63$ & 5.55 & $51.81 \pm 5.66$ & 51.19 \\
\hline C & $101.67 \pm 10.89$ & 100 & $5.67 \pm 0.58$ & 5.58 & $45.77 \pm 5.68$ & 45.02 & $5.59 \pm 0.68$ & 5.50 & $50.24 \pm 5.54$ & 49.41 \\
\hline B & $110.75 \pm 10.91$ & 100 & $4.98 \pm 0.65$ & 4.50 & $48.25 \pm 5.47$ & 43.57 & $6.04 \pm 1.56$ & 5.45 & $57.52 \pm 5.21$ & 51.94 \\
\hline $\mathrm{L}$ & $85.29 \pm 7.89$ & 100 & $4.12 \pm 0.53$ & 4.83 & $34.65 \pm 5.17$ & 40.63 & $5.09 \pm 1.94$ & 5.97 & $46.52 \pm 3.48$ & 54.54 \\
\hline$\overline{\bar{x}}$ & 99.72 & 100 & 4.81 & 4.83 & 43.40 & 43.52 & 5.59 & 5.62 & 51.52 & 51.77 \\
\hline $\mathrm{DP}$ & \pm 10.58 & - & \pm 0.68 & \pm 0.01 & \pm 6.00 & \pm 0.02 & \pm 0.39 & \pm 0.00 & \pm 4.57 & \pm 0.02 \\
\hline
\end{tabular}

Quadro 4. Média $(\bar{X})$ e respectivos desvios padrão (DP) de cada uma das componentes cronométricas da prova dos 200m livres, para a categoria G4/G5 feminino. TTP - tempo total de prova; TP - tempo de partida; TV - tempo de viragem; TChg - tempo de chegada; TN - tempo de nado; \% - percentagem relativa do tempo total que os nadadores demoram a cumprir determinada componente da prova.

\begin{tabular}{|c|c|c|c|c|c|c|c|c|c|c|}
\hline \multicolumn{11}{|c|}{ G4/G5 - FEMININO } \\
\hline Prova & TTP & & TP & & TV & & TChg & & TN & \\
\hline $200 \mathrm{~m}$ & $\overline{\mathrm{X}} \pm \mathrm{DP}$ & $\%$ & $\overline{\mathrm{X}} \pm \mathrm{DP}$ & $\%$ & $\overline{\mathrm{X}} \pm \mathrm{DP}$ & $\%$ & $\overline{\mathrm{X}} \pm \mathrm{DP}$ & $\%$ & $\overline{\mathrm{X}} \pm \mathrm{DP}$ & $\%$ \\
\hline Livres & $\begin{array}{l}196.47 \\
\pm 16.20\end{array}$ & 100 & $\begin{array}{c}4.15 \\
\pm 0.34\end{array}$ & 2.11 & $\begin{array}{l}94.03 \\
\pm 7.68\end{array}$ & 47.86 & $\begin{array}{r}5.39 \\
\pm 0.71\end{array}$ & 2.74 & $\begin{array}{l}89.28 \\
\pm 7.94\end{array}$ & 45.44 \\
\hline
\end{tabular}

Quadro 5. Média $[\bar{X}]$ e respectivos desvios padrão (DP) de cada uma das componentes cronométricas da prova dos $400 \mathrm{~m}$ livres, para a categoria $\mathrm{G} 4 / \mathrm{G} 5$ feminino. TTP - tempo total de prova; TP - tempo de partida; TV - tempo de viragem; TChg - tempo de chegada; TN - tempo de nado; \% - percentagem relativa do tempo total que os nadadores demoram a cumprir determinada componente da prova.

\begin{tabular}{|c|c|c|c|c|c|c|c|c|c|c|}
\hline \multicolumn{11}{|c|}{ G4/G5 - FEMININO } \\
\hline Prova & TTP & & TP & & TV & & TChg & & TN & \\
\hline $400 \mathrm{~m}$ & $\overline{\mathrm{X}} \pm \mathrm{DP}$ & $\%$ & $\bar{X} \pm D P$ & $\%$ & $\bar{X} \pm D P$ & $\%$ & $\overline{\mathrm{X}} \pm \mathrm{DP}$ & $\%$ & $\overline{\mathrm{X}} \pm \mathrm{DP}$ & $\%$ \\
\hline Livres & $\begin{array}{l}378.85 \\
\pm 31.72\end{array}$ & 100 & $\begin{array}{c}3.92 \\
\pm 0.52\end{array}$ & 1.03 & $\begin{array}{l}210.37 \\
\pm 20.12\end{array}$ & 55.53 & $\begin{array}{c}4.91 \\
\pm 0.80\end{array}$ & 1.30 & $\begin{array}{l}164.56 \\
\pm 12.65\end{array}$ & 43.44 \\
\hline
\end{tabular}

Variáveis Dependentes: Tempo de Partida, Tempo de Nado, Tempo de Viragem, Tempo de Chegada. Variável Independente: Tempo Total de Prova.

O estudo apresentou algumas limitações com relação a idade e a colecta de dados no que se refere ao tamanho da piscina. Assim, a pesquisa foi realizada para um determinado grupo etário, a recolha de dados se deu em piscina de 25,0 x 12,5 x 2,0m. Neste sentido, os resultados se limitam inicialmente ao factor etário e a predições em piscinas curtas.

\section{APRESENTAÇÃO DOS RESULTADOS}

De seguida, vamos apresentar o perfil das provas do G4/G5 feminino (Quadros 3, 4 e 5).

$\mathrm{Na}$ prova dos $100 \mathrm{~m}$ nas diferentes técnicas, o comportamento dos parâmetros de tendência central confirma os valores descritos na literatura, nomeadamente a importância relativa do TN e do TV e um menor contributo relativo do TChg. e TP respectivamente (Quadro 4). Na prova dos 200 m livres, verificou-se que o aumento na distância de prova se tra- 
duziu, comparativamente às provas mais curtas, numa maior duração no TN e nas competências motoras associadas à viragem (Quadro 5).

Tal como já havíamos observado na prova dos $200 \mathrm{~m}$ livres também aqui na prova dos $400 \mathrm{~m}$ livres se acentua o crescente contributo relativo nas competências motoras associadas às viragens e uma diminuição relativamente uniforme nas restantes componentes. As viragens apresentam uma acção diferenciada, uma vez que estão condicionadas à visualização da borda da piscina, noção de espaço e uma acção motora diferenciada dos gestos cíclicos presentes na natação pura desportiva.
De seguida, apresentaremos o perfil das provas da categoria G5/G6 masculino (Quadros 6, 7 e 8). A distribuição relativa do contributo das diferentes componentes nos nadadores evidencia a mesma tendência já observada para as nadadoras (Quadro 7). Tal como já havíamos verificado para o género feminino, existe uma prevalência relativa da TV face às restantes componentes em estudo para a prova dos 200 livres (quadro 7) e 400 livres (Quadro 8).

Também o caso dos nadadores demonstra a importância relativa que o domínio específico das componentes motoras associadas às viragens parece ter na prestação destes sujeitos (Quadro 8).

Quadro 6. Média $(\bar{x})$ e respectivos desvios padrão (DP) de cada uma das componentes cronométricas da prova dos $100 \mathrm{~m}$ mariposa, costas, bruços e livres; para a categoria G5/G6 masculino. M - mariposa; C - costas; $B$ - bruços; $L$ - livres; TTP - tempo total de prova; TP - tempo de partida; TV - tempo de viragem; TChg tempo de chegada; $T N$ - tempo de nado; \% - percentagem relativa do tempo total que os nadadores demoram a cumprir determinada componente da prova.

\begin{tabular}{|c|c|c|c|c|c|c|c|c|c|c|}
\hline \multicolumn{11}{|c|}{ G5/G6 - MASCULINO } \\
\hline Prova & TTP & & TP & & TV & & TChg & & TN & \\
\hline $100 \mathrm{~m}$ & $\bar{X} \pm D P$ & $\%$ & $\overline{\mathrm{X}} \pm \mathrm{DP}$ & $\%$ & $\overline{\mathrm{X}} \pm \mathrm{DP}$ & $\%$ & $\bar{X} \pm D P$ & $\%$ & $\overline{\mathrm{X}} \pm \mathrm{DP}$ & $\%$ \\
\hline $\bar{M}$ & $93.03 \pm 8.87$ & 100 & $4.05 \pm 0.47$ & 4.35 & $41.66 \pm 4.53$ & 44.78 & $5.35 \pm 0.56$ & 5.75 & $47.32 \pm 5.11$ & 50.87 \\
\hline C & $94.72 \pm 8.92$ & 100 & $5.20 \pm 0.63$ & 5.49 & $41.35 \pm 4.24$ & 43.65 & $5.45 \pm 1.37$ & 5.75 & $48.17 \pm 4.73$ & 50.86 \\
\hline$B$ & $104.78 \pm 9.46$ & 100 & $4.48 \pm 0.52$ & 4.28 & $45.87 \pm 4.40$ & 43.78 & $5.68 \pm 0.72$ & 5.42 & $54.43 \pm 5.43$ & 51.95 \\
\hline L & $80.40 \pm 7.33$ & 100 & $3.84 \pm 0.43$ & 4.78 & $36.07 \pm 6.22$ & 44.86 & $4.56 \pm 1.31$ & 5.67 & $41.47 \pm 4.19$ & 51.58 \\
\hline$x$ & 93.23 & 100 & 4.39 & 4.73 & 41.24 & 44.27 & 5.26 & 5.65 & 47.85 & 51.32 \\
\hline $\mathrm{DP}$ & \pm 10 & \pm 0.0 & \pm 0.60 & \pm 0.01 & \pm 4.01 & \pm 0.64 & \pm 0.49 & \pm 0.00 & \pm 5.30 & \pm 0.01 \\
\hline
\end{tabular}

Quadro 7. Média $(\bar{x}$ ) e respectivos desvios padrão (DP) de cada uma das componentes cronométricas da prova dos 200m livres, para a categoria G5/G6 masculino. TTP - tempo total de prova; TP - tempo de partida; TV - tempo de viragem; TChg - tempo de chegada; TN - tempo de nado; \% - percentagem relativa do tempo total que os nadadores demoram a cumprir determinada fase da prova.

G5/G6 - MASCULINO

\begin{tabular}{|c|c|c|c|c|c|c|c|c|c|c|}
\hline Prova & TTP & & TP & & TV & & TChg & & TN & \\
\hline $200 \mathrm{~m}$ & $\overline{\mathrm{X}} \pm \mathrm{DP}$ & $\%$ & $\overline{\mathrm{X}} \pm \mathrm{DP}$ & $\%$ & $\overline{\mathrm{X}} \pm \mathrm{DP}$ & $\%$ & $\overline{\mathrm{X}} \pm \mathrm{DP}$ & $\%$ & $\overline{\mathrm{X}} \pm \mathrm{DP}$ & $\%$ \\
\hline Livres & $\begin{array}{l}188.14 \\
\pm 9.05\end{array}$ & 100 & $\begin{array}{c}4.29 \\
\pm 0.14\end{array}$ & 2.28 & $\begin{array}{c}89.95 \\
\pm 4.90\end{array}$ & 47.81 & $\begin{array}{c}5.21 \\
\pm 0.45\end{array}$ & 2.77 & $\begin{array}{r}85.3 \\
\pm 3.92\end{array}$ & 45.34 \\
\hline
\end{tabular}

Quadro 8. Média $(\bar{x}$ ] e respectivos desvios padrão (DP) de cada uma das componentes cronométricas da prova dos 400m livres, para a categoria G5/G6 masculino. TTP - tempo total de prova; TP - tempo de partida; TV - tempo de viragem; TChg - tempo de chegada; TN - tempo de nado; \% - percentagem relativa do tempo total que os nadadores demoram a cumprir determinada fase da prova.

\begin{tabular}{|c|c|c|c|c|c|c|c|c|c|c|}
\hline \multicolumn{11}{|c|}{ G5/G6 - MASCULINO } \\
\hline Prova & TTP & & TP & & TV & & TChg & & TN & \\
\hline $400 \mathrm{~m}$ & $\bar{X} \pm D P$ & $\%$ & $\overline{\mathrm{X}} \pm \mathrm{DP}$ & $\%$ & $\overline{\mathrm{X}} \pm \mathrm{DP}$ & $\%$ & $\overline{\mathrm{X}} \pm \mathrm{DP}$ & $\%$ & $\overline{\mathrm{X}} \pm \mathrm{DP}$ & $\%$ \\
\hline Livres & $\begin{array}{l}360.74 \\
\pm 21.18\end{array}$ & 100 & $\begin{array}{c}3.66 \\
\pm 0.37\end{array}$ & 1.01 & $\begin{array}{l}205.50 \\
\pm 24.03\end{array}$ & 56.97 & $\begin{array}{c}4.67 \\
\pm 0.67\end{array}$ & 1.29 & $\begin{array}{l}151.58 \\
\pm 17.75\end{array}$ & 42.02 \\
\hline
\end{tabular}


Na perspectiva da determinação das equações de regressão linear das componentes TP, TV, TChg e TN nas diferentes provas, fomos calcular os valores de $\mathrm{A}$ (declive) e de B (ordenada na origem) através de procedimentos estatísticos inferenciais.

Nos quadros que se seguem (Quadros 9, 10 e 11) encontram-se descritos os valores de A e de B, e o valor do erro relativo de cada uma das componentes da prova, para o G4/G5 feminino e G5/G6 masculi- no, relativamente às provas de $100 \mathrm{~m}$ (mariposa, costas, bruços e livres), $200 \mathrm{~m}$ e $400 \mathrm{~m}$ livres. $\mathrm{Na}$ teoria da probabilidade e na estatística, a variância de uma variável aleatória é uma medida da sua dispersão estatística, indicando quão longe em geral os seus valores se encontram do valor esperado. A variância é o valor esperado do quadrado do desvio de $X$ da sua própria média (Quadro 12).

Quadro 9. Valores de $A$ (declive) e de B (ordenada na origem] para cada uma das componentes da prova 100 m (mariposa, costas, bruços e livres); e respectivo erro relativo para categoria de cadetes - G4/G5 femininos e G5/G6 masculinos. TTP - tempo total de prova; TP - tempo de partida; TV - tempo de viragem; TChg tempo de chegada; TN - tempo de nado.

\begin{tabular}{cccccccc}
\hline $\begin{array}{l}\text { Prova } \\
100 \mathrm{~m}\end{array}$ & Componentes & \multicolumn{2}{c}{ MASCULINO } & & FEMININO & B & Erro relativo \\
\hline M & A & 0.0329 & 0.989 & $9.27 \%$ & 0.04957 & -0.566 & $5.66 \%$ \\
& TP & 0.445 & 0.267 & $5.45 \%$ & 0.427 & 1.718 & $5.76 \%$ \\
& TV & 0.0517 & 0.544 & $6.09 \%$ & 0.0474 & 0.826 & $7.12 \%$ \\
& TChg & 0.523 & -1.297 & $4.68 \%$ & 0.523 & -1.151 & $2.38 \%$ \\
\hline C & TN & 0.0529 & 0.189 & $8.11 \%$ & 0.04346 & 1.249 & $6.09 \%$ \\
& TP & 0.448 & -1.047 & $3.47 \%$ & 0.487 & -3.730 & $4.51 \%$ \\
& TV & 0.07667 & -1.814 & $22.08 \%$ & 0.0455 & 0.967 & $8.41 \%$ \\
& TChg & 0.499 & 0.858 & $3.36 \%$ & 0.470 & 2.481 & $4.26 \%$ \\
\hline B & TN & 0.0473 & -0.475 & $5.77 \%$ & 0.0473 & -0.260 & $8.06 \%$ \\
& TP & 0.418 & 2.114 & $4.27 \%$ & 0.491 & -6.084 & $2.53 \%$ \\
& TV & 0.05 & 0.438 & $9.64 \%$ & 0.0618 & -0.808 & $23.51 \%$ \\
& TChg & 0.535 & -1.639 & $3.64 \%$ & 0.462 & 6.344 & $2.28 \%$ \\
\hline TN & TP & 0.04194 & 0.466 & $7.81 \%$ & 0.04939 & -0.0913 & $8.9 \%$ \\
& TV & 0.347 & 8.182 & $2.69 \%$ & 0.200 & -5.511 & $6.06 \%$ \\
& TChg & 0.09186 & -2.825 & $25.04 \%$ & 0.127 & -5.726 & $33.08 \%$ \\
& TN & 0.557 & -3.266 & $2.41 \%$ & 0.350 & 16.622 & $10.31 \%$ \\
\hline
\end{tabular}

Quadro 10. Valores de A (declive) e de B (ordenada na origem) para cada uma das componentes da prova $200 \mathrm{~m}$ livres, e respectivo erro relativo para a categoria de cadetes - G4/G5 femininos e G5/G6 masculinos. TTP - tempo total de prova; TP - tempo de partida; TV - tempo de viragem; TChg - tempo de chegada; TN tempo de nado.

\begin{tabular}{|c|c|c|c|c|c|c|c|}
\hline \multirow{2}{*}{$\begin{array}{l}\text { Prova } \\
200 \mathrm{~m}\end{array}$} & \multirow[b]{2}{*}{ Variáveis } & \multicolumn{2}{|c|}{ MASCULINO } & \multicolumn{4}{|c|}{ FEMININO } \\
\hline & & A & B & Erro Relativo & A & B & Erro relativo \\
\hline \multirow[t]{4}{*}{ Livres } & TP & 0.00918 & 2.56 & $2.65 \%$ & 0.00961 & 2.257 & $7.29 \%$ \\
\hline & TV & 0.514 & -6.684 & $1.60 \%$ & 0.466 & 2.489 & $1.39 \%$ \\
\hline & TChg & 0.0375 & -1.835 & $5.98 \%$ & 0.03498 & -1.478 & $8.08 \%$ \\
\hline & TN & 0.398 & 10.486 & $1.89 \%$ & 0.482 & -5.384 & $1.67 \%$ \\
\hline
\end{tabular}


Quadro 11. Valores de $A$ (declive) e de B (ordenada na origem) para cada uma das componentes da prova $400 \mathrm{~m}$ livres, e respectivo erro relativo para a categoria de cadetes - G4/G5 femininos e G5/G6 masculinos. TTP - tempo total de prova; TP - tempo de partida; TV - tempo de viragem; TChg - tempo de chegada; TN tempo de nado.

\begin{tabular}{cccccccc}
\hline $\begin{array}{l}\text { Prova } \\
\text { 400m }\end{array}$ & Componentes & A MASCULINO & B & Erro Relativo & A & BEMININO & Erro relativo \\
\hline Livres & TP & 0.009639 & 0.186 & $8.55 \%$ & 0.01405 & -1.4 & $6.85 \%$ \\
& TV & 0.792 & -80.19 & $8.52 \%$ & 0.616 & -23.049 & $2.30 \%$ \\
& TChg & 0.02072 & -2.8 & $10.94 \%$ & 0.0154 & -0.921 & $13.02 \%$ \\
& TN & 0.198 & 80.005 & $11.57 \%$ & 0.370 & 24.449 & $2.91 \%$ \\
\hline
\end{tabular}

Quadro 12. Percentagem das variâncias de cada uma das componentes da prova dos 100 metros (mariposa, costas, bruços e livres) e 200 e 400 metros livres, para cada um dos géneros ( $\mathbf{q}$ - género feminino, - género masculino); e respectivas diferenças entre os géneros ( $\mathbf{q} / \mathbf{3}$ ). TTP - tempo total de prova; TP tempo de partida; TV - tempo de viragem; TChg - tempo de chegada; TN - tempo de nado.

\begin{tabular}{|c|c|c|c|c|c|c|c|c|c|c|c|c|c|}
\hline Prova & Téc. & $\begin{array}{l}\text { TP } \\
\mathbf{9}\end{array}$ & 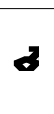 & $\begin{array}{c}\neq \\
(\boldsymbol{q} / \boldsymbol{\sigma})\end{array}$ & $\begin{array}{l}\text { TV } \\
\mathbf{q}\end{array}$ & $d$ & $\begin{array}{c}\neq \\
(\boldsymbol{q} / \boldsymbol{\sigma})\end{array}$ & $\begin{array}{c}\text { TChg } \\
\mathbf{9}\end{array}$ & $d$ & $\begin{array}{c}\neq \\
(\boldsymbol{q} / \boldsymbol{\theta})\end{array}$ & $\begin{array}{l}\text { TN } \\
\mathbf{q}\end{array}$ & $d$ & $\begin{array}{c}\neq \\
(\boldsymbol{q} / \boldsymbol{d})\end{array}$ \\
\hline \multirow[t]{4}{*}{$100 \mathrm{~m}$} & $M$ & 81 & 36 & 45 & 92 & 75 & 17 & 60 & 66 & $6 \%$ & 95 & 81 & 14 \\
\hline & $C$ & 65 & 55 & 10 & 87 & 89 & 2 & 52 & 23 & 29 & 85 & 88 & 3 \\
\hline & B & 62 & 75 & 13 & 95 & 80 & 15 & 17 & 42 & 25 & 94 & 87 & 7 \\
\hline & $L$ & 52 & 51 & 2 & 84 & 91 & $?$ & 25 & 24 & 1 & 62 & 94 & 32 \\
\hline $200 \mathrm{~m}$ & $L$ & 19 & 32 & 12 & 97 & 92 & 5 & 62 & 53 & 10 & 97 & 83 & 13 \\
\hline $400 \mathrm{~m}$ & $\mathrm{~L}$ & 73 & 28 & 45 & 94 & 47 & 47 & 36 & 41 & 6 & 86 & 2 & 83 \\
\hline
\end{tabular}

Na prova dos 100 m mariposa, o TTP está mais condicionado ao $\mathrm{TN}$, com $95 \%$ e $81 \%$, para os géneros feminino e masculino respectivamente e grupos (G4/G5 e G5/G6).

Em relação à prova dos $100 \mathrm{~m}$ costas, também para ambos os géneros (feminino e masculino) e grupos (G4/G5 e G5/G6) é o TV com $87 \%$ e $89 \%$ do TTP a variável que melhor explica o TTP. De referir ainda que a percentagem do TChg assume uma importância substancialmente diferente em cada um dos géneros. Para o G4/G5 feminino, 52\% do TTP é explicado pelo TChg, enquanto que para o G5/G6 masculino, o TCgh representa apenas $23 \%$ do TTP. Relativamente aos $100 \mathrm{~m}$ bruços, verifica-se uma diferença entre os dois géneros relativamente à importância do TChg. Para as provas do grupo feminino, $17 \%$ do TTP é explicado por esta componente. Em contrapartida, para as provas do grupo masculino, a percentagem explicativa do TChg. no TTP é de $42 \%$. Ainda respeitante a esta prova, a variável que explica melhor o TTP para o G4/G5 é o TV e para o G5/G6 é o TN com $95 \%$ e $87 \%$, respectivamente.
De todas as provas, é nos 100m livres que o G5/G6 obteve uma percentagem maior nas variáveis explicativas do TTP. Verifica-se que $94 \%$ do TTP é explicado pelo $\mathrm{TN}$, o que significa que esta variável assume grande importância nas provas masculinas. No grupo feminino, a variável que melhor explica o TTP é o TV (84\%).

Ainda com base no quadro 12 , podemos verificar que para o G4/G5 feminino, na prova dos $200 \mathrm{~m}$ livres, $97 \%$ do TTP é explicado pela variável TV. Ainda na mesma prova, a variável que melhor explica o TTP para o G5/G6 masculino é também o TV, o qual assume uma importância igualmente elevada, $92 \%$.

Por último, temos a prova dos $400 \mathrm{~m}$ livres, na qual existe uma maior discrepância entre os valores do G4/G5 e do G5/G6. Em ambos os grupos, é o TV que melhor explica o TTP. No entanto, para o grupo feminino, a percentagem é bastante maior, 94\%, enquanto que para o grupo masculino, o TV apenas consegue explicar $47 \%$ do TTP. De referir ainda que as percentagens explicativas do TP e do TN entre os géneros são bastante diferentes, sendo que no 
G4/G5 as percentagens são bastante mais significativas. No que respeita ainda aos $400 \mathrm{~m}$ livres femininos, $86 \%$ do TTP é explicado pelo TN, enquanto que para o género masculino, a mesma variável explica apenas $2,4 \%$ do TTP.

\section{Exemplo concreto para a definição de objectivos}

Este tópico tem por objectivo apresentar um exemplo prático relativo a uma situação hipotética - preparação de um nadador para uma competição importante. A prova a que nos reportamos será a dos 100 $\mathrm{m}$ livres e os tempos que aqui apresentamos referem-se a um caso real de uma nadadora, que acompanhamos ao longo das nossas filmagens.

Assim, no seguimento da metodologia preconizada, a primeira etapa será a de se proceder a uma análise diagnóstico relativamente às diferentes componentes da prova, padrão que se quer programar, face aos valores reais de uma nadadora pertencente à amostra de estudo (Quadro 13).
Quadro 13. Tempos absolutos efectivos que uma nadadora da categoria de cadetes - G4/G5 femininos - apresentou na prova de $100 \mathrm{~m}$ livres. TTP tempo total de prova; TP - tempo de partida; TV - tempo de viragem; TChg tempo de chegada; $T N$ - tempo de nado.

\begin{tabular}{ccccc}
\hline $\begin{array}{c}\text { TTP } \\
{[\mathbf{s}]}\end{array}$ & $\begin{array}{c}\text { TP } \\
{[\mathbf{s}]}\end{array}$ & $\begin{array}{c}\text { TV } \\
{[\mathbf{s}]}\end{array}$ & $\begin{array}{c}\text { TChg } \\
\text { [s] }\end{array}$ & $\begin{array}{c}\text { TN } \\
{[\mathbf{s}]}\end{array}$ \\
\hline 72,57 & 3,07 & 28,24 & 3,81 & 37,45 \\
\hline
\end{tabular}

A segunda etapa a ser efectuada é a de, com base nos valores de A e de B obtidos, e com base no objectivo de prestação da nadadora em questão (TTP de 68 segundos), definir os tempos preditos para cada uma das componentes da prova (Quadro 14). A terceira e última etapa será a de com base na análise comparativa entre os valores reais e os valores preditos da prestação objectivo (TTP de 68 segundos) definir as componentes prioritárias a desenvolver (Quadro 15).

\begin{abstract}
Quadro 14. Tempos (em segundos] a realizar pela nadadora da categoria cadete - G4/G5 femininos, na prova de $100 \mathrm{~m}$ livres, nas diferentes componentes de prova, de acordo com a aplicação das equações de regressão. TTP - tempo total de prova; TP - tempo de partida; TVs - tempo das viragens; TChg - tempo de chegada; s-segundos.
\end{abstract}

\begin{tabular}{|c|c|c|}
\hline G4/G5 - Feminino & & \\
\hline Valores de A e B & Desenvolvimento das equações de regressão & \\
\hline Determinados para a categoria de Cadetes & (2a etapa) & \\
\hline $\mathrm{TP}=0,04939 \times \mathrm{TTP}+(-0,0913)$ & $\mathrm{TP}=0,04939 * 68+(-0,0913)$ & $3,26 \mathrm{~s}$ \\
\hline$T V=0,600 x \operatorname{TP}+(-16,531)$ & $\mathrm{TV}=0,600 * 68+(-16,531)$ & $24,26 \mathrm{~s}$ \\
\hline TChg $=0,127 \times \mathrm{TTP}+(-5,726)$ & TChg $=0,127 * 68+(-5,756)$ & $2,88 \mathrm{~s}$ \\
\hline $\mathrm{TN}=0,350 \times \mathrm{TTP}+16,622$ & $\mathrm{TN}=0,350 * 68+16,622$ & $37,58 \mathrm{~s}$ \\
\hline & TTP $=68$ segundos & \\
\hline
\end{tabular}

Quadro 15. Comparação entre os valores referentes ao processo de definição de objectivos e os valores obtidos na avaliação diagnóstico pela nadadora da categoria cadete - G4/G5 femininos, na prova de 100 m livres, nas diferentes componentes de prova, de acordo com a aplicação das equações de regressão. TTP tempo total de prova; TP - tempo de partida; TVs - tempo das viragens; TChg - tempo de chegada; s - segundos.

\begin{tabular}{lcccc}
\hline $\mathbf{1 0 0}$ metros Livres - G4/G5 Feminino & & & \\
\hline Componentes [s] & Modelo Real & Modelo Ideal & Diferenças & Componentes Prioritárias a Trabalhar \\
\hline TP & 3,07 & 3,26 & $-0,19$ & $3^{\text {a }}$ \\
TV & 28,24 & 24,26 & $+3,98$ & $1^{\text {a }}$ \\
TChg & 3,81 & 2,88 & $+0,93$ & $2^{\text {a }}$ \\
TN & 37,45 & 37,58 & $-0,13$ & $4^{\text {a }}$ \\
TTP & 72,57 & 67,98 & $+4,59$ & \\
\hline
\end{tabular}


Com base nos dados, podemos verificar que o modelo real não está muito desfasado do modelo ideal. A nadadora possui um tempo de partida e de nado inferior ao do modelo ideal. Tal acontece, porque utilizamos a melhor nadadora para efectuar a demonstração. Contudo, através deste exemplo, sabemos que alguns aspectos devem ser melhorados durante o treino, ou seja, sabemos que a viragem e a chegada são as componentes prioritárias a serem trabalhadas.

\section{DISCUSSÃO DOS RESULTADOS}

Tendo em consideração que os objectivos deste trabalho se centraram: (i) na determinação das expressões preditivas para as diferentes variáveis cronométricas, tendo em conta o conhecimento do TTP para os 400 e os 200 metros livres e para os 100 metros mariposa, costas, bruços e livres; por género e por prova; (ii) no desenvolvimento das normativas para cada uma das variáveis cronométricas estudadas, por género e por prova; (iii) e na operacionalização de um modelo para a definição de objectivos orientado para a prestação, podemos verificar que os resultados demonstraram diferenças entre as diversas provas e distâncias estudas, havendo uma variação da importância das diferentes componentes de nado de acordo com a prova, o género e a técnica de nado. Quando efectuámos uma comparação entre as várias provas estudadas e analisamos os valores expressos nos Quadros 3, 4, 5, 6, 7 e 8, verificou-se que à medida que a distância de prova aumenta a importância do TP diminuiu enquanto que a do TV aumenta. Estes dados vêm confirmar os resultados obtidos por diferentes autores $(1,4,15,31,32)$, que afirmam que com o aumento da distância de prova, é previsível a ocorrência de uma diminuição da importância do TP e, inversamente, um aumento da importância relativa do somatório do TV.

A par desta situação, verificou-se também uma diminuição percentual da importância da chegada, à medida que a distância de prova aumenta, o que vai ao encontro das conclusões retiradas do estudo sobre a variabilidade entre as variáveis cronométricas preconizadas por Arellano (4). No entanto, o valor percentual para cada uma das variáveis é diferente, o que é perfeitamente normal, já que os autores realizaram os seus estudos a partir de provas disputadas em piscinas de 50 metros $(22,27)$. Como este estudo foi elaborado numa piscina de 25 metros, os valores obtidos para o TN são claramente inferiores e os obtidos para o TV são claramente superiores, comparando com os resultados publicados pelos autores acima referenciados (9).

$\mathrm{Na}$ análise comparativa entre o G4/G5 feminino e o G5/G6 masculino, verificámos que os parâmetros TP, TChg, TN e TV são consideravelmente inferiores nos nadadores quando comparados com as nadadoras. Isto talvez possa ser explicado pelo facto da amostra apresentar uma idade em que o nível maturacional tende a ser diferenciado com repercussões nas características antropométricas $(12,17)$.

No entanto, não se verifica o mesmo para o TP na prova dos 200 metros livres e para o TV na prova dos 100 metros livres. Estes dados não são totalmente concordantes com a análise pormenorizada efectuada por Arellano et al. (5) nos Jogos Olímpicos de Barcelona, uma vez que demonstraram que as mesmas variáveis, incluindo também a distância por ciclo, são significativamente maiores em homens do que em mulheres. Também o estudo de Arellano et al. (6), realizado com os nadadores que participaram no dia olímpicos da juventude em 2001, concluiu que os nadadores do género masculino são mais rápidos em cada uma das fases da prova, e demonstraram um maior índice de nado e distância por ciclo, enquanto que a frequência gestual é idêntica. A diferença do TP em cada um dos géneros é, em média, de 1 segundo para as provas de 100 metros. O mesmo acontece no estudo realizado por Arellano et al. (6), realizado com nadadores cujas idades estavam compreendidas entre os 15 os 16 anos, e com nadadoras com 13 e 14 anos.

A diferença do TV entre os dois géneros para a prova de 100 metros mariposa é de 1,08 segundos, para a prova dos $100 \mathrm{~m}$ costas é de 1,48 segundos, para a prova dos $100 \mathrm{~m}$ bruços é de 0,82 segundos, e para a prova dos $100 \mathrm{~m}$ livres é de 0,14 segundos. Os dados do estudo realizado por Arellano et al. (6) demonstram que a diferença entre o TV para os dois géneros, nas provas dos $100 \mathrm{~m}$ mariposa e bruços, é de 1,4 segundos, e nas provas de $100 \mathrm{~m}$ costas e livres é de 1 segundo.

Relativamente aos coeficientes de correlação e às equações de predição lineares obtidas no nosso estudo, podemos desde já referir que não temos termo 
de comparação, uma vez que não foram encontrados estudos realizados para esta categoria de nadadores. Podemos analisar os dados obtidos pelo estudo realizado por Arellano et al. (5). No entanto receamos que não seja uma comparação muito válida, uma vez que a nossa amostra é de uma categoria bastante mais jovem e, para além disso, os autores analisaram apenas provas de 50 e 100 metros livres, realizadas numa piscina de 50 metros. Por tudo isto, julgamos que se compreende o facto de as diferenças serem notórias.

O desenvolvimento da equação 1 , para a prova dos 100 metros livres, permitiu obter para os dois géneros os seguintes valores de cada uma das componentes da prova.

Quadro 16. Valores de $A$ [declive] e $B$ (ordenada na origem], para as diferentes componentes de prova dos dois géneros na prova de $100 \mathrm{~m}$ livres, em nadadores da categoria de cadetes - G4/G5 femininos e G5/G6 masculinos. TTP - tempo total de prova; TP - tempo de partida; TV - tempo de viragem; TChg - tempo de chegada; $T N$ - tempo de nado.

\begin{tabular}{cc}
\hline G4/G5 - Feminino & G5/G6 Masculino \\
\hline Tempo médio efectivo [s] & Tempo médio efectivo $[\mathbf{s}$ ] \\
\hline TP $=4.81$ & TP $=4.39$ \\
TV $=43.40$ & TV $=41.24$ \\
TChg $=5.59$ & TChg $=5.26$ \\
TN $=51.52$ & TN $=47.85$ \\
\hline
\end{tabular}

Para além das diferenças existentes entre o estudo de Arellano et al. (6) e o nosso relativamente ao nível prestativo dos nadadores da amostra, as quais já foram acima referenciadas, convém mencionar ainda que a metodologia relativa aos procedimentos para captação e registo de imagens foi diferente, atendendo a que se tratava de uma piscina de $50 \mathrm{~m}$ (marcas de referência utilizadas foram: (i) o TP foi medido aos $10 \mathrm{~m}$, (ii) a marca de referência para calcular o TV foi de 7,5m, (iii) o TChg foi medido aos $10 \mathrm{~m}$. Em relação ao objectivo geral deste trabalho, podemos concluir que todas as variáveis cronométricas obtiveram relações estatisticamente significativas com o TTP e foram estabelecidas as respectivas equações de predição de rendimento. Deste modo, com a elaboração deste estudo, pensamos ter contribuído para o desenvolvimento da natação em Portugal, uma vez que tornamos possível aos treina- dores saberem quais as componentes de prova que deverão em cada momento ser prioritariamente trabalhadas, bem como a modelação da prestação esperada para os mesmos nadadores. Esta tarefa foi conseguida tendo como base não só o tempo final da prova, mas também o tempo de cada uma das variáveis constituintes da mesma. Segundo Arellano (4), o treinador deve elaborar o modelo de competição mais conveniente para cada um dos seus nadadores, fazendo adaptações baseadas nas características individuais de cada um. A análise das componentes da prova durante a época ajudará a monitorizar o plano de treino, e a fazer as respectivas adaptações necessárias ao modelo de treino proposto.

Tal como foi apresentado no modelo para a definição de objectivos orientado para a prestação, concluímos que este modelo oferece algumas vantagens para o planeamento do treino, pois permite aos treinadores saberem se os objectivos parciais e gerais para a prova em questão foram conseguidos ou não. Permite-lhes ainda saber quais as etapas prioritárias a serem trabalhadas, para que se possa atingir o objectivo previamente estabelecido. Com o nosso exemplo, podemos concluir ainda que a nadadora precisa de melhorar a execução das viragens e da chegada (26).

Os resultados apurados no decurso do presente estudo permitem-nos o estabelecimento ainda das seguintes conclusões:

(i) Verificou-se, para a generalidade dos parâmetros estudados, variação entre géneros;

(ii) Com o aumento da distância de prova houve uma diminuição da importância relativa do TP e, inversamente, um aumento da importância relativa do TV. Assim, é indiscutível a influência que a melhoria de qualquer um destes segmentos de prova pode ter sobre a prestação final, tal como corrobora com outros estudos $(2,22,26)$. Aliás, os resultados sugerem-nos que do ponto de vista do modelo habitual de preparação utilizado para esta categoria de nadadores, provavelmente os técnicos deverão dar maior ênfase a este segmento de prova.

Relativamente ainda a este parâmetro, concluiu-se também que, a prova dos 100 metros livres é a que apresenta partidas mais rápidas, tanto no G4/G5 feminino como no G5/G6 masculino;

(iii) O estilo de bruços é aquele em que o tempo de viragem é superior; 
(iv) As provas de maior distância (200 e 400 metros) apresentam uma percentagem de nado menor, enquanto que as provas de 100 metros (livres e bruços) são as que apresentam a percentagem mais elevada. Estes dados parecem-nos importantes, na medida em que nos levam a crer que os nadadores passam mais tempo a nadar nas provas de $100 \mathrm{~m}$ (livres e bruços), do que nas provas dos $200 \mathrm{e}$ $400 \mathrm{~m}$ livres. Assim, pensamos que durante o processo de treino dos nadadores, se deve dar mais ênfase à técnica de nado nas provas de distância mais curta. Por outro lado, nas provas de maior distância o treino das viragens deve assumir maior relevância (8); (v) Foi ainda possível verificar que, para todas as provas à excepção do TP nos $200 \mathrm{~m}$ livres e do TV nos $100 \mathrm{~m}$ livres, os nadadores do G5/G6 masculino são mais rápidos a partir, a virar e a chegar do que as nadadoras pertencentes ao G4/G5.

Tendo como base as conclusões apresentadas, este estudo tende a apresentar-se como uma boa forma de predizer os tempos de cada uma das componentes da prova, para as faixas etárias, géneros sexuais e provas realizadas em piscina curta $(25 \mathrm{~m})$, não generalizáveis para outras condições uma vez que a prestação depende de uma multiplicidade de factores (3, $19,30)$.

\section{CORRESPONDÊNCIA}

\section{António José Silva}

Universidade de Trás-os-Montes e Alto Douro, Departamento de Desporto CIFOP

Rua Dr. Manuel Cardona 5000 Vila Real, Portugal

e-mail:ajsilva@utad.pt 


\section{BIBLIOGRAFIA}

1. Alves F (1989). Bases mecânicas da natação desportiva. Manual do curso de treinadores de II grau. Lisboa: Federação Portuguesa de Natação.

2. Alves F, Lopes A, Ribeiro JP (1991). Análise da competição em natação desportiva. Natação 4 (14): 8-12.

3. Alves JGB (2003). Physical activity in chilhood: promoting adult health. Rev Bras Saúde Mat Inf 3(1):5-6.

4. Arellano R (1993). El análisis cinemática de la competición: su utilización en el entrenamiento. Madrid: Federación Española de Natación, Escola Nacional de Entrenadores.

5. Arellano R, Brown P, Cappaert J, Nelson RC (1996). Application of regression equations in the analysis of 50 and $100 \mathrm{~m}$ swimming races of 1992 Olympic games. In: João Abrantes (eds). XIV International Symposium on Biomechanics in Sports. Edições FMH, Universidade Técnica de Lisboa, 274-277.

6. Arellano R, Sánchez-Molina J, Navarro F, Aymerich J (s.d.). Analysis of 100-M backstroke, breastroke, butterfly and freestyle swimmers at the 2001 European Youth Olympic Days. Madrid: Federación Española de Natación.

7. Barbosa TM, Fernandes R, Keskinen KL, Colaço P, Cardoso C, Silva J, Vilas-Boas JP. (2006) Evaluation of the energy expenditure in competitive swimming strokes. Int J Sports Med 27(11):894-9

8. Blanksby B, Nicholson L, Elliott B. (2002) Biomechanical analysis of the grab, track and handle swimming starts: an intervention study. Sports Biomech 1(1): 11-24.

9. Blanksby B, Skender S, Elliott B, McElroy K, Landers G (2004) An analysis of the rollover backstroke turn by agegroup swimmers. Sports Biomech 3(1):1-14.

10. Costill DL, Kovaleski J, Porter D, Kirwan J, Fielding, R, King, D (1985). Energy expenditure during front crawl swimming: predicting success in middle-distance events. Int. J Sports Med 6: 266-270.

11. Deleaval P (1990). Un autre regard sur la performance. EPS 21:49-53.

12. Duarte MFS (1993). Physical Maturation: A review with special reference to brazilian children. Cad Saúde Públ 9(supl. 1):71-84.

13. Craig A, Skehan P, Pawelczyk JE, Boomer W (1985). Velocity, stroke rate and distance per stroke during elite swimming competition. Med Sci Sports Exerc 17(6): 625-634.

14. Haljand R, Saagpakk R (1994). Swimming competition analysis of the European Sprint Swimming Championship. Stavanger: LEN.

15. Hay J (1988). The status of research on the biomechanics of swimming. In: Ungerechts B, Wilke K, Reischle K (eds.). Swimming Science $V$. Illinois: Human Kinetics Publishers, 3-14.

16. Hay JG, Guimarães A, Grimston SK (1983). A quantitative look at swimming biomechanics. Swimming Technique 20(2): 11-17.

17. Hue O, Galy O, Blonc S, Hertogh C. (2006) Anthropometrical and physiological determinants of performance in French West Indian monofin swimmers: a first approach. Int J Sports Med 27(8):605-9
18. Martinez R (1990). Colóquio. Europa Júniores Dunkerque 90 - Análise Técnica: Projecto nacional (I parte). Revista Natação 12: 14-17.

19. Michaud PA, Suris JC (2005). Sports activity during adolescence: a challenge for health professionals and for the society. Rev Med Suisse 1(28):1835-6, 1838-9.

20. Osborne JW (2000). Prediction in multiple regression. Practical Assessment. Research \& Evaluation 7 (2). $h t t p: / /$ pareonline.net/getvn.asp? $v=7 \& n=2$.

21. Reischle K (1993). Biomecánica de la Natación. Madrid: Gymnos Editorial.

22. Seifert L, Boulesteix L, Carter M, Chollet D. (2005) The spatial-temporal and coordinative structures in elite male 100-m front crawl swimmers. Int J Sports Med 26(4):286-93

23. Smith D, Cappaert J, Curry I, Heest J, Kranenburg K, Kwon Y, Lefort J, Luy B, Maki B, Mason B, Norris S (1996). Competition analyses of swimming events. Olympic Games - Atlanta 1996. IOC Sub commission on Biomechanics and Physiology of Sport.

24. Stefani RT. (2006) The relative power output and relative lean body mass of World and Olympic male and female champions with implications for gender equity. $J$ Sports Sci 24(12):1329-39

25. Toubekis AG, Douda HT, Tokmakidis SP. (2005) Influence of different rest intervals during active or passive recovery on repeated sprint swimming performance. Eur J Appl Physiol 93(5-6):694-700

26. Tourny-Chollet C, Chollet D, Hogie S, Papparodopoulos C. (2002) Kinematic analysis of butterfly turns of international and national swimmers. J Sports Sci 20(5):38390

27. Trinity JD, Pahnke MD, Reese EC, Coyle EF. (2006) Maximal mechanical power during a taper in elite swimmers. Med Sci Sports Exerc 38(9):1643-9

28. Verjoshanski L (2001). Teoría e metodología del entrenamiento deportivo. Barcelona: Ed. Paidotribo.

29. Vicente L (2002). Análisis de la competición y entrenamiento. XXII Congresso AETN. Córdoba: AETN.

30. Vicent-Rodriguez G, Ara I, Perez-Gomez J, Dorado C, Calbet JÁ (2005). Muscular development and physical activity as major detrerminants of femoral bone mass acquisition during growth. Br J Sports Med 39(9):611-6.

31. Vilas-Boas JP (1992). O índice de braçada como critério para a avaliação da técnica de bruços. Not FPNatação 5 (17): 4-9.

32. Wakayoshi K, Nomura T, Takahashi G, Mutoh Y, Miyashito M. (1992). Analysis of swimming races in the 1989 Pan Pacific swimming championships and Japanese Olympic trials. In: MacLaren D, Reilly T (eds). Biomechanics and Medicine in Swimming. Swimming Science VI. London: E \& FN Spoon, 135-141.

33. Williams SJ, Kendall LR. (2006) A profile of sports science research (1983-2003). J Sci Med Sport [doi:10.1016/j.jsams.2006.07.016; Epub ahead of print] 